\title{
CALCULATIONS OF NUCLEAR REACTIONS PROBABILITY IN A CRYSTAL LATTICE OF LANTHANUM DEUTERIDE
}

\author{
V.A. KIRKINSKII AND YU. A. NOVIKOV \\ Institute of Mineralogy and Petrography, \\ Siberian Branch of the Russian Academy of Sciences, \\ Prospect Acad. Koptyuga, 3, Novosibirsk, 630090, Russia, \\ E-mail: kirkinsk@uiggm.nsc.ru
}

\begin{abstract}
The dynamic model of electron orbitals deformation (EODD) was previously devised for palladium deuteride. It has now been applied to calculate the probability of nuclear reactions of hydrogen isotopes in the crystal lattice of lanthanum deuteride. In a series of computer simulations, the probability of D-D approach for random initial conditions was calculated, when the initial energies of the approaching deuterons were set in the range of $0.001-9.0 \mathrm{eV}$. For each experimental value of D-D approach the reaction rate was calculated on the shifted Coulomb potential with the shift energy, which equals to the energy of screening. The mean distance of D-D approach on the whole series equals $0.19 \AA$. More than $54 \%$ of all experimental values show an approach of deuterons for a distance less than $0.1 \AA$. The average reaction rate for the given set of the initial conditions is $10^{3.28}$ $\mathrm{DD}^{-1} \mathrm{~s}^{-1}$. This is three orders of magnitude less than the analogous rate calculated earlier for palladium deuteride. Allowing for the higher $\mathrm{D}$ content and the higher number of adjacent tetrahedral sites in $\mathrm{LaD}_{3}$ as compared with $\mathrm{PdD}_{0,6}$, an overall nuclear fusion rate in lanthanum deuteride will be only slight lower than in palladium deuteride, that is, $10^{-12}-10^{-14} \mathrm{DD}^{-1} \mathrm{~s}^{-1}$.
\end{abstract}

To achieve a more sophisticated understanding of the mechanism and peculiar features of nuclear reactions in condensed matter, it is very important to expand a circle of substances that have been investigated. However, direct experimental research of new materials involves considerable difficulties, as the observable effects only slightly exceed the sensitivity of instrumentation, or the background level. In this connection, a random search of all substances to find which ones can catalyze cold nuclear fusion would be labor consuming and ineffective, especially taking into account the variations of their physical state and impurity content. Although most research on nuclear reactions in metal have been done with palladium, the high price and limited quantities of this material hinder the practical use of it. From the foregoing, the importance of theoretical calculation for search of materials is clear - a search to find which materials can be activated to produce nuclear fusion at low energies.

We conducted a simulation of nuclear reactions of deuterium in palladium crystal lattice based on a dynamic model of electron orbital deformation in which hydrogen atom are located in octahedral sites of closely packed metal atoms [1-4]. The calculation results correspond within an order of magnitude to the experimental measurements of excess energy output (see, for example, [5] and reviews in [1,6]).

Later, using the same model, the rate of a nuclear reaction was calculated in a titanium deuteride, where deuterium atoms are situated in tetrahedral sites of a crystal lattice, and the limiting content corresponds to $\mathrm{TiD}_{2}$ crystal structure [4]. 
In the present paper we calculate the probability of a nuclear interaction in lanthanum deuteride, in which deuterium atoms can occur both in octahedral and tetrahedral sites of closely packed lanthanum atoms forming the $\mathrm{LaD}_{3}$ crystal lattice. The high content of deuterium in lattice should be favorable for implementing a nuclear interaction. Studies of lanthanum deuteride are also valuable because based on them we can draw conclusions about analogous compounds of lanthanide elements and yttrium, which have a similar lattice with slightly diminished crystal lattice parameters.

For calculations of probability of nuclear reactions of hydrogen isotopes in the crystal lattice of lanthanum deuteride the dynamic model of electron orbitals deformation (EODD) offered earlier for palladium deuteride was used [1-3]. The hydrogen isotopes predominantly occupy tetrahedral sites in the face-centered cubic lattice of lanthanum hydride. Symmetrical positions of deuterium atoms concerning the edge connecting two adjacent tetrahedral sites in lanthanum were selected as initial conditions for numerical tests. In a series of computer simulations, the probability of D-D approach for random initial conditions was calculated in the range of initial energies of approaching deuterons 0.001-9.0 eV (the potential barrier for diffusion of deuterium atoms in lanthanum is about $0.43 \mathrm{eV}$ ). The most favorable conditions of two deuterons approaching one another were determined by preliminary computer modeling [1] within the framework of the described model to reduce the calculation time. For each experimental value of D-D approach the reaction rate was calculated on the shifted Coulomb potential with the shift energy, which is equal to the energy of screening.

The series consists of 40000 experimental values. The mean distance of D-D approach on the whole series equals $0.19 \AA$, however, more than $54 \%$ of all experimental values show an approach of deuterons for a distance less than $0.1 \AA$. If one considers the reaction rate at each case of approach, and then averages as a whole on the entire sample, the general reaction rate for the given set of the initial conditions will make $10^{3.28} \mathrm{DD}^{-1} \mathrm{~s}^{-}$ ${ }^{1}$. It is 3 orders of magnitude less than the analogous rate calculated earlier for palladium deuteride [1-3], but more than ten times higher than the rate calculated for titanium deuteride [4].

In order to investigate the influence of the initial outer electron orbital radius on a efficiency of deuteron approaching, another set of simulations was carried out. In the second series the range of outer orbital radii $(a-0.4) \pm 0.05 \AA$ was used instead of $(a-0.2) \pm 0.05 \AA$ in the fist series (were $a$ is a half of distances between lanthanum atoms located in the vertex of tetrahedral site). The results of the second series essentially differ from the first series ones. The mean distance of D-D approach on the series equals 0.25 A. 


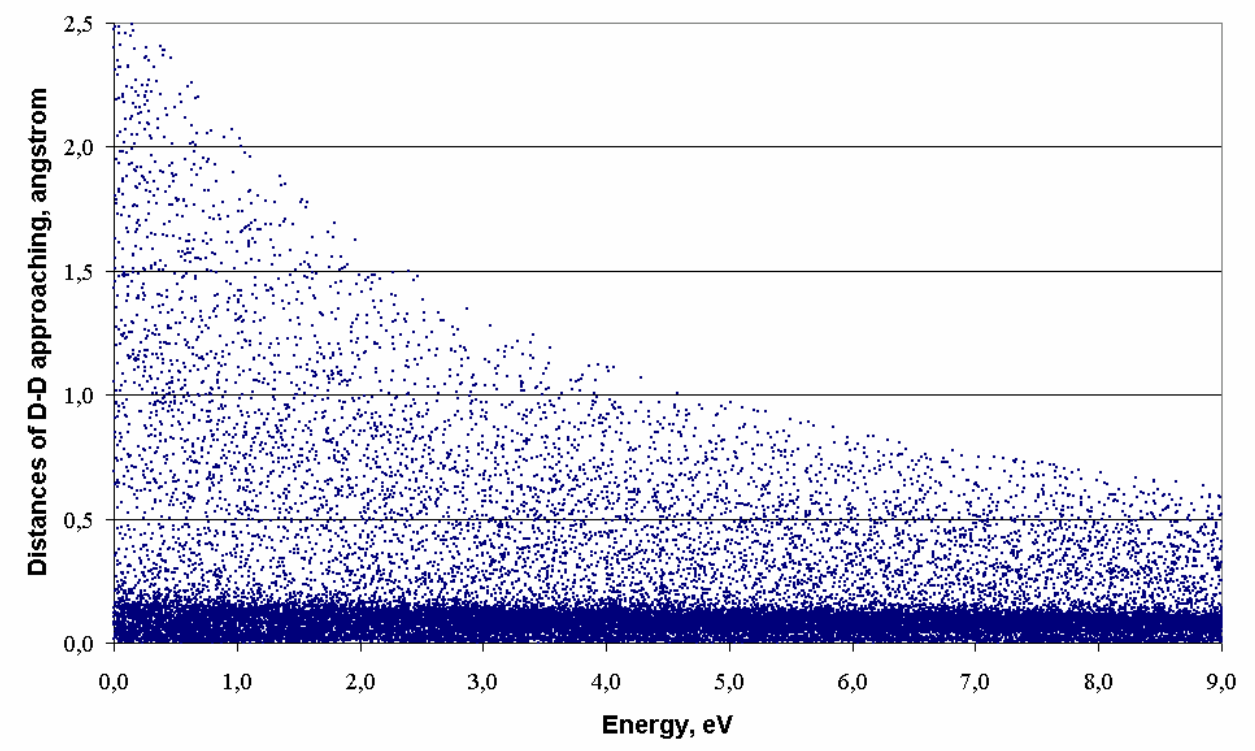

Figure 1. Distribution of minimal distances of D-D approach during their counter motions on the boundary of the neighbor tetrahedral sites in lanthanum deuteride vs their started kinetic energy.

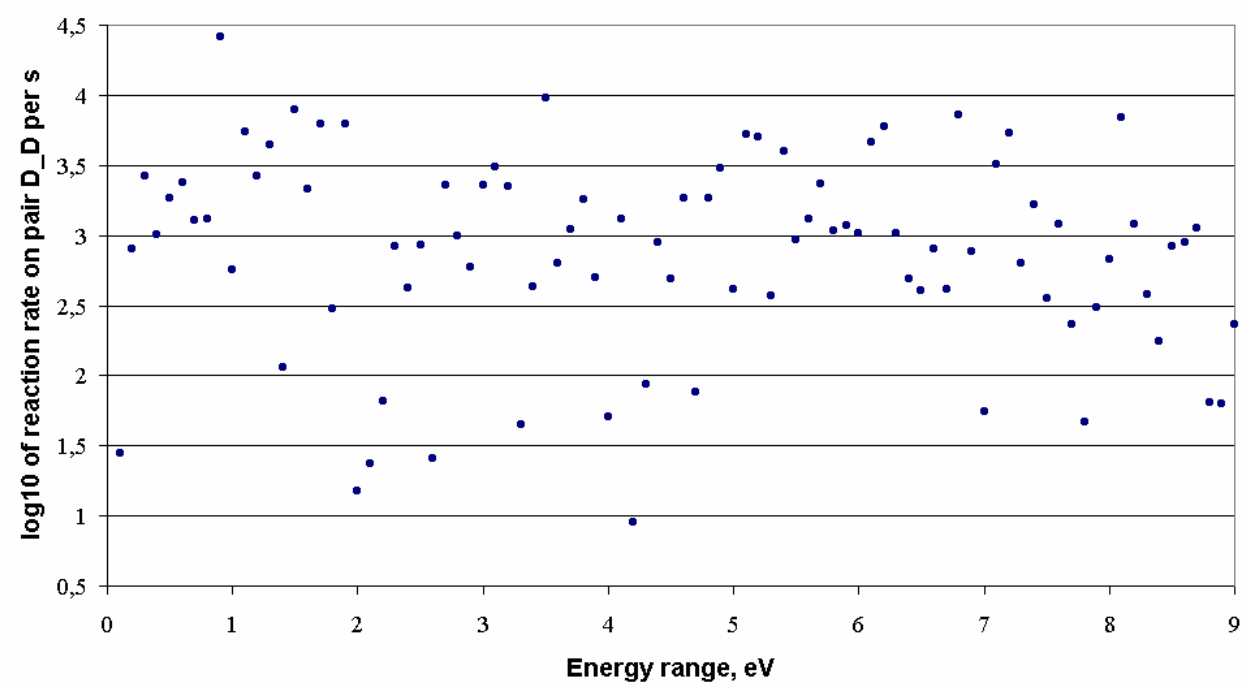

Figure 2. Energy dependence of D-D reaction rate in lanthanum deuteride according EODD simulations for the first series. 


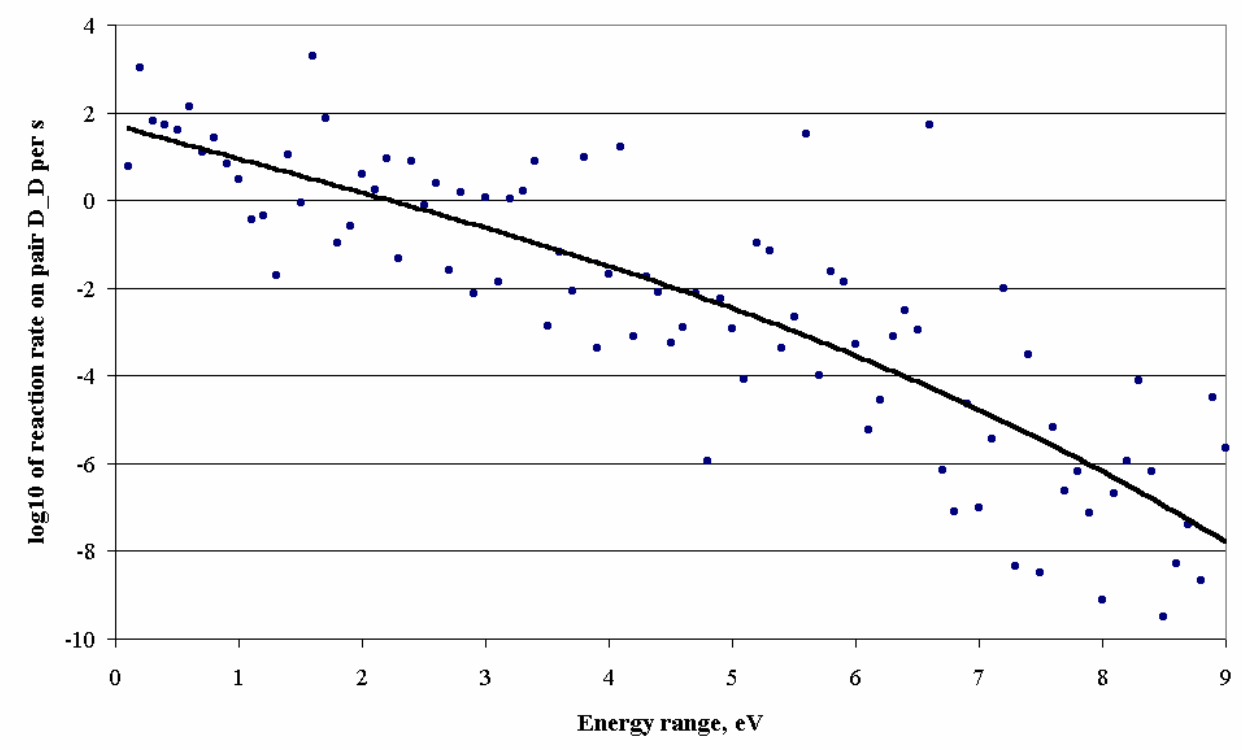

Figure 3. Energy dependence of D-D reaction rate in lanthanum deuteride according EODD simulations for the second series.

More than 33\% of all experimental values show an approach of deuterons at a distance less than $0.1 \AA$. If one considers the reaction rate at each case of approach, and then one averages as a whole over the entire sample, the average reaction rate for the given set of the initial conditions will be $10^{1.41} \mathrm{DD}^{-1} \mathrm{~s}^{-1}$. Comparing fig. 2 and fig. 3 one can see that at the energy $<1 \mathrm{eV}$ the reaction rates in both cases are nearly equal, but at higher energy the dependence character differ. The D-D reaction rate in lanthanum deuteride have a reverse dependence on energy in comparison with palladium deuteride [see 1-3].

To optimize of calculations the most favorable initial conditions were selected. To obtain the true results, calculated values should be also be multiplied by a correction factor [1], which allows for the probability of the occurrence of these favorable conditions. When calculating this factor it is necessary to take into consideration the fact that the content of deuterium in lanthanum deuteride is 6 times higher than in palladium deuteride and the number of deuterium atoms in adjacent tetrahedral sites is 32 times higher than in adjacent octahedral sites in $\mathrm{PdD}_{0.5}$. Besides, in $\mathrm{LaD}_{3}$ there are closelyspaced adjacent octahedral and tetrahedral sites occupied by deuterium. Thus, if it is able to provide high mobility of deuterons in $\mathrm{LaD}_{3}$ crystal lattice, the reaction rate will be only slight lower than the rate calculated earlier [1-3] and experimentally observed in palladium deuteride (see, for example, [5] and reviews in [1,6]).

Owing to the diminished parameters of crystal lattices of deuterides of other rare earth metals and yttrium, the reaction rate of a nuclear fusion in them can be closely matched to the rate in palladium deuteride, i.e. $10^{-12}-10^{-14} \mathrm{DD}^{-1} \mathrm{~s}^{-1}$. Since the cost of a 
natural mixture of rare earth metals is much lower than the same weight of palladium, and rare earth metals are much more abundant in nature than palladium (and other platinum group metals), they are attractive perspective catalysts for nuclear fusion at low energies.

This work was supported by the RFBR (grant No 05-05-64930).

\section{References}

1. V.A. Kirkinskii and Yu.A. Novikov, Theoretical modeling of cold fusion. Novosibirsk, Novosibirsk State University, 2002, 105 p.

2. V.A. Kirkinskii and Yu.A. Novikov, In the book: "Condensed Matter Nuclear Science" (Proceedings of the ICCF-9, ed. by Xing Z. Li), 162 (2003).

3. V.A. Kirkinskii and Yu.A. Novikov, Europhys. Lett. 67, 362 (2004).

4. V.A. Kirkinskii and Yu.A. Novikov, Calculations of Nuclear Reactions Probability in a Crystal Lattice of Titanium Deuteride, in the book: "Condensed Matter Nuclear Science. Proceedings of the ICCF-10", ed. by. P. Hagelstein and S. Chubb (2005).

5. V.A. Kirkinskii, V.A. Drebushchak and A.I. Khmelnikov, Europhys. Lett. 58, 462 (2002).

6. P. Hagelstein et al. New Physical Effects in Metal Deuterides (http://www.lenrcanr.org/acrobat/Hagelsteinnewphysica.pdf). 